\title{
Editorial
}

\section{Guy Haworth, Chess, PuyoPuyo and the Computer Olympiad 2020}

Tristan Cazenave

LAMSADE, Université Paris-Dauphine, PSL, CNRS, Paris, France

E-mail: Tristan.Cazenave@dauphine.psl.eu

The ICGA lost a great contributor to the journal and to the computer chess world with the passing of Guy Haworth in June. Jaap van den Herik and Mark Winands publish in this issue A tribute to Guy Haworth. It recalls the numerous contributions that Guy Haworth made to our community. Thanks to his wife Annette, we could recover the last contribution from Guy Haworth, Chess without Draws that is published in this issue. It reports a proposal by 'Aloril' defining a logical sequence of increasingly subtle and decreasingly rewarded 'winning' goals. The proposal is called 'Mobility Chess' and can reduce and even eliminate the incidence of draws.

The second scientific contribution is On the power of lookahead in single-player PuyoPuyo by Yasuhiko Takenaga, Sho Kikuchi and Hushan Quan. PuyoPuyo is a Tetris-type game. The paper addresses the impact of lookahead of input pieces on winning strategies of the single player game.

In this issue we also have reports on last year Computer Olympiad. A report by Hiroyuki Iida, Jonathan Schaeffer and I-Chen Wu entitled The Computer Olympiad 2020 and a report by Quentin CohenSolal and Tristan Cazenave that gives a description of their Deep Reinforcement Learning algorithm which participated in the Computer Olympiad 2020 entitled DESCENT wins five gold medals at the Computer Olympiad.

In the news section you will find the announcement of the 2021 Advances in Computer Games conference and the SSDF Chess Engine Rating List.

Tristan Cazenave 